\title{
Research on the Application of Software Agent in Command and Display system
}

\author{
Duan Huifen ${ }^{1, a}$ 1, Bao Junlei ${ }^{1, b}$, Zhang Dehua ${ }^{1, b}$, Li Lei $^{1, b}$ \\ ${ }^{1}$ Satellite Marine Tracking and Control Department of China, Jiangyin, 214431, China \\ adhf4321@126.com.cn, b5 5ublic@126.com
}

Keywords: Software Agent, Command and Display, Control body

\begin{abstract}
In the aerospace tracking and control mission, the command and display system is deployed to a large number of different command terminals that located in different locations, which makes the coordination between these systems become difficult. In this paper, an agent-based control system is proposed. Using software agent technology, the operational semantics of agent is defined, and the remote control between the controller and the functional terminals is realized, which solved the coordinating problem among different command and display modules with different functions, so as to improve the reliability and efficiency of the control system .
\end{abstract}

\section{Introduction}

Space flight mission is a very large and complex real-time operating task, which involves a large number of equipments and personnel, and the command and coordination system is very complex. In order to ensure the task is operating with strict order, an advanced mission command and monitoring system is indispensible. The command and display system can collect, process and display the information from the whole mission to the commanders and engineers. This system uses distributed structure, thus, in the implementation of task, we need coordinate the contents, display styles and timing among different display workstations. With the development of the system scale, the difficulty of the coordination is becoming greater. In the past, the coordination mainly rely on post staff according to the scheduling instructions for software operation control, which has a low efficiency, and easy to make mistakes. In this paper system, using the agent technology is introduced to solve the distributed collaborative working between the different terminal software modules of the different terminals, so as to optimize the operation process, and ensure the quality of the task.

\section{Software Agent Technology}

Agent is generally referred to the certain intelligence and autonomy, which can respond to changes in the environment and the corresponding processing software system [1-3]. With regard to the definition of agents, researchers in different fields have different understandings of them. According to their own needs, the researchers put forward different explanations to the agency. The definition of FIPA (Intelligent Agent Foundation) is defined as follow: the agent is a basic actor in a field. It has one or more of the tasks and performance as a consistent and complete implementation of the model, and it can interact with external software, operators, and communication facilities. [4, 5]

The agent has the following characteristics [6]:

(1) With the autonomous ability and autonomously execute;

(2) It communicate information (possibly directly or indirectly) through an agent communication language with other agents or operators;

(3) It can sense the change of the working environment and make the response.

The agent software can replace the executor to perform the entrusted task, and communicate with other software agents to complete the task [7], .According to the software agent mobility, the agent can be divided into dynamic and static proxy agent [7-9]. The agent of the paper is static agent that resides on a host, perform a specific task and wait for the user delegation request the execution of the 
work, and to exchange information to communicate with the outside world, also hand over to another agent.

\section{Agent Application}

Role of the Agent. In the command and display system of the testing task, the command display software is designed as the control body, the agent and the function body are two other kinds of objects. The instance of the control body is the user interface, which is used to generate the control command, collecting feedback information, monitoring the system operation. An instance of the proxy is in the terminal of each function body, which is used to receive the command issued by the control body, the analysis of control commands, is responsible for the transmission of the function, the activation of the corresponding processing module. In the same time, the control body responsees to the control body. The function body is a software system which is used in the system, each function body and agent has the same communication interface. The agent is responsible for the transfer, explanation and control of the command between the control body and the function body in the command display.

Analysis of the Application. According to the analysis of the command and display business, four kinds of command sets are designed for software agents, which are task preparation, monitoring control, time control, and agent communication. The task is prepared to include an agent environment parameter setting, which is used to specify the location of the proxy function body and the position of the control body. The task automatic process configuration, according to the task of actual combat plan, on time order to define the control process, the task does not require manual intervention, the agent according to the process of automatic control of the operation of the various functions. The software interface configuration, control body in preparation tasks based on the database of the processing object format description information and generates the display function of data interface, when there is a change in interface through a proxy distribution to all the display function, ensure the consistency of the interface changes. The command set maintenance, the command set is the rule base of the agent system, when the system has new control functions need to be extended, a new rule can be added to the rule base.

The monitoring control is divided into public control class, special control class. The public control class that is suitable for all the functions of the rules, including system start, exit, reset system, operation and return to the control rules. The special control to apply only to certain functions of the control rules, including communication function of transmission control, control panel and data processing function of network control and record library control, track control calculation, display interface upgrade, the screen switches, and so.

The time control system is divided into system time comparison, emergency time points. The system time comparison is based on command system to carry out a unified time, the terminal time machine to bring the bias correction. The feature point emergency time class is for the task of feature points in time often marks the phase of the beginning or the end of the task, timely and accurate received the characteristic point is particularly important. The feature point time includes the departure time, the separation time of the star (ship) and the time of the separation of the track, so as to ensure that the system can receive the time of the feature points.

The agent communication includes the communication between agent and control body or the communication between agent and function body. The communication between the agent and the control body is mainly completed by the transmission of the command from the remote to the local terminal. The communication between the agent and the function body is mainly completed in the transmission of the local terminal. By using agent technology, the integration of remote control and display system, workflow process and control interface are realized. 


\section{The Agent Realization}

Agent Structure.In the command display system, we design the agent for three layer structure, as the figure 1 shows. The control body transmits the control command to the agent through the transport layer, the proxy receives the remote control command through the transport layer. By using the rules in the rule base, the control commands received by the analytic layer are resolved, and the functional body which needs to be activated is determined through the interface layer.

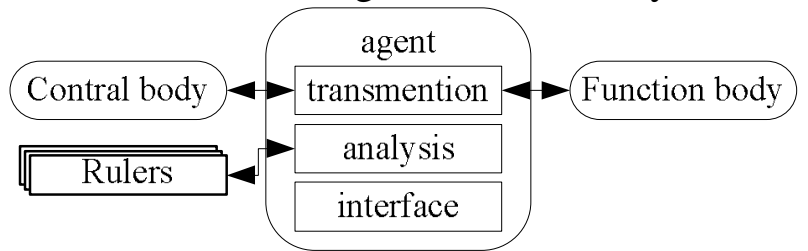

Fig.1 Agent structure

Remote Control. The command and monitoring system of the testing task designed a control, centralized control, four kinds of function data communications, data processing, data display, and 3D simulation. In order to ensure the reliability of the system, data communication, data processing were set up the main, the preparation of two functional. Data display settings of the large screen projection display, command and dispatch display, display the chief seats, station remote display some data display function. As shown in figure 2, each function of the system is controlled by the unified management of the agent receiving control center.

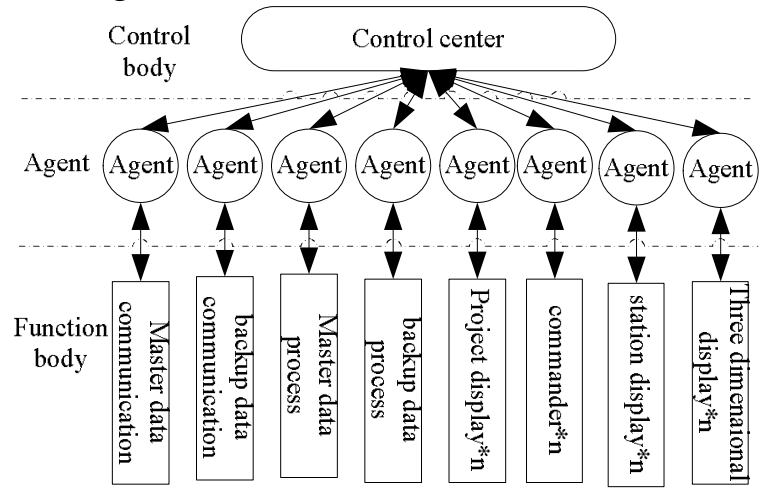

Fig.2 System composition

In the task preparation stage, the control center according to the configuration database task table to generate new interface control document, agents collect function interface file version, feedback to the control center, when the interface file version of the function body is lower than the center of the interface file version control, the control center will be the interface file distribution to the agent, the agent responsible for the completion of the update function interface, to ensure the consistency of the system interface. When the interface changes, the control center will change the interface file version number, every function of the system in the operation of the interface can be completed through the agent update. At the stage of the task operation, through the control center user interface, according to the single instruction form the dispatching command to the agent, the feedback information of the agency are collected, and then sent back.

According to the characteristics of the command, the communication of the control center and the agent is realized by two ways: UDP and TCP/IP. TCP/IP is used to control the individual command of each agent, send a specific command and status to a function body.UDP group broadcast is used in the entire control of the general control of the field of radio and state reception.

The communication of the agent and the function uses memory mapping and message mechanism combining method. The memory mapping is a process of a virtual address is mapped to a file, the other process can share the virtual address, and is also a local inter process communication way. The Win32 API sharing (shared memory) memory is actually the mapping file a special case. The implementation method of the agent and the control center and the function body communication is shown in Figure3. 


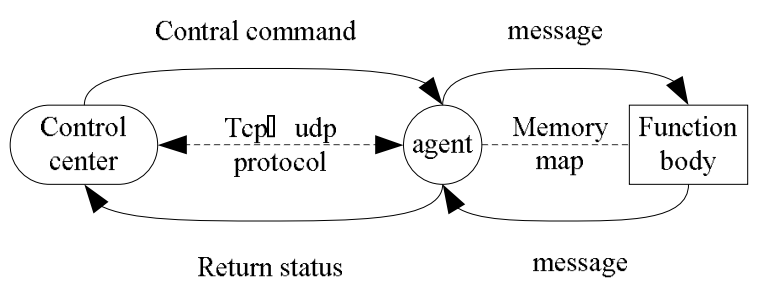

Fig.3 Communication implementation of proxy

With this way of communication, on the one hand the loosely coupling between the agent and the function of the body is realized, and any party to a separate program failure, does not affect to the other party program processing. On the other hand, because the memory mapped files are independent to the agent and the function body, so as long as there is party programs do not shut down, the data will not be lost, which makes software controlled even restart, and still continue to access the stored data in the file mapping. This design method not only realizes the control of the function body, but also does not affect the original logic relation and the business process flow.

Workflow Process. At the stage of the task operation, in addition to the man-machine interface can be controlled by the operator, and an automatic way, the control center according to process tasks to develop the work order by the way of time. The command sent to the agent automatically by the program, the function body responds automatically according to the commands received by the agent, and the supporting work flow is controlled by the program.

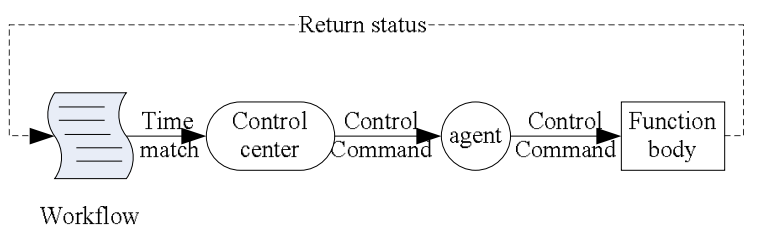

Fig. 4 Workflow process

Control Interface Standardization.In the command and monitoring system of test task, the functional bodies that interact with the agent are different. (Fig. 2), each function's command is not the same. In order to facilitate the expansion and configuration of the system, the command in the rule base of the rapid positioning in the design for the agent defined a unified interface format, mapping address and rules library. When creating a file mapping object, the agent uses the 0xFFFFFFFF file handle, and the process uses the 0xFFFFFFFF to replace the file handle.

We define a unified interface element for the agent, which including: check code, command code, command parameter 1 , and command parameter 2 .The check code is used to identify the agency received orders to avoid system error. The command code is used to transfer the control command, the command parameter is used to indicate the state of the command and the state of the feedback state after the command is executed. Acting is according to the command code to parse out the meaning of the command, and usingthe message to inform the function of the implementation of the corresponding command and feedback the results of the implementation.

In the software implementation, the following simple structure is used to define the agent communication interface:

typedef __noge struct SendCommand

\{ int checkout;// check code

int CommandCode;// command name

_int64 Wparam;// Command parameter 1

int Lparam;// Command parameter 2

\}SendCommand;

The following structure is used to define the interface of the agent:

typedef public __value struct SqlStatus // Local structure matching analysis for \{

String*CodeCn; // Command Chinese name int Wparam;// command name 
\}SqlStatus;

_int64 Lparam;// command parameters

int Label;// label

The agent through the command to achieve the control of the function, the functional experience will command the state after the implementation of information through the agent to inform the control body. In the command and monitoring system of test task, according to the system control requirements, the specific command set and the state set are designed, constitute the rule base of the agent together, such as the system has new requirements, you can add a new command to the rule base. Table 1 gives the definition of the command set; table 2 gives the definition of the state set.

Table 1 Command set definition

\begin{tabular}{|c|c|c|c|}
\hline category & Command code & content & Param \\
\hline \multirow{9}{*}{ System command } & 1000 & Control center is set up & \\
\hline & 1001 & Boot process switch & 1: start 0: stop \\
\hline & 1002 & Program start switch & 1: start 0: stop \\
\hline & 1003 & System timing & TICKS of DATETIME \\
\hline & 1004 & System reset & \\
\hline & 1005 & Request status return & \\
\hline & 1006 & Fire up time T0 & TICKS of DATETIME \\
\hline & 1007 & separation point time & TICKS of DATETIME \\
\hline & 1008 & Return point time & TICKS of DATETIME \\
\hline \multirow{3}{*}{$\begin{array}{l}\text { processing } \\
\text { command }\end{array}$} & 1200 & Send data switch & 1: start $0:$ stop \\
\hline & 1201 & write database switch & 1: start $0:$ stop \\
\hline & 1202 & $\begin{array}{l}\text { Transmit trajectory data } \\
\text { switch }\end{array}$ & 1:real data 0 :theory data \\
\hline Display command & 1300 & Display the specified picture & picture Number \\
\hline
\end{tabular}

The message mechanism can be used at the same time, because the data processing, monitor and display real-time requirements, processor overhead larger. In order to reduce software controlled access to shared memory processor overhead, using the message notification, the notification process from the memory mapped files in order to obtain the command.

\section{Conclusions}

In the command and monitoring system of aerospace tracking and control task, we use proxy mechanism to control to realize the loose coupling between the body and the function body, ensuring the tasks to perform reliably and reducing the operation coordination between different functions. also In addition the number of post operation personnel and the error rate of the operation are also reduced. The proposed system achieved good effect in the practical applications. The application of agent technology can also provides a reference method for the establishment of software remote support services.

\section{References}

[1] Xu Hongquan, Zhang Luqin, Zhan Guangpin: Research on software Agent technology and software architecture[J] Computer \& Digital Engineering, 2009,37(5):59-62

[2] Chen Xiaoxiang: Research on Component Technique and Software Agent[J]Computer Security, 2009(2):70-73

[3]MAO Xin-jun, CHANG Zhi-ming: A gent-Oriented Software Design Patterns[J] Computer Engineering \& Science,2011,33(6):72-78

[4]FIPS Specification:fipa9713[EB/OL],http://www.fipa.org/spcc/,1999; 
[5]MA Qiangming:Design and Implementation of Collaboration Supply Chain Mamagement System Base On Mutil-agent[D].Chen Du: University Electronic Science and Technology of CHINA,2012 [6]WU Di,YAN He,CAI Wei:Research and Application on Agent-based Learning Resource Discovery Technology[J] Computer Science, 2009,36(4):185-188

[7]CHEN Qiang,WEI Chen-jian:Design of a Distributed Intelligent E-Commerce System Based on Multi Agents[J] Computer Engineering \& Science, 2010,32(7):143-146

[8]LI Linglin,TAN Jinpeng:Application research of personalized process model[J]Technology Development of Enterprise ,2014,33(1):1-3

[9]Dong Menggao:Research on Agent-based Running Mechanism and Implementation Technologies for Self-Adaptive Systems[D] Chang Sha: National University of Defense Technology 2011 